Garstang: In those objects in which several iron lines have been seen the relative intensities seem to agree tolerably well with our calculated values. Even if you can only measure the strongest line with acceptable accuracy that would be valuable and probably sufficient for an ionic abundance determination.

Osterbrock: Higher stages of ionization, namely [FeX] $\lambda 6374$ and [FeXI] $\lambda 7892$ are clearly present in several Seyfert galaxies. It would be worthwhile to look carefully for these lines in high-ionization planetaries, particularly NGC 7027 because they furnish information on the input radiation field at very high energies.

Garstang: I agree, but one should notice that according to our simple calculations on ionic abundances of iron there is a wide range of ionizing radiation temperatures for which much of the iron is in the FeVIII and FeIX stages, so that a rather high temperature would be required to produce FeX.

Nussbaumer: Collision strengths and transition probabilities for the Fe ions can be calculated, but to what accuracy? We calculate at present FeVI data, the collision strengths by the Distorted Wave method. The differences between the results of the two calculations may give a hint about uncertainties associated with such calculations.

Garstang: There are many uncertainties in the calculations, and it is indeed difficult to make an assessment of what the uncertainties are. An independent calculation would help considerably in this respect. Improvements in the photoionization cross-sections are also of great importance in trying to improve iron abundance determinations.

THE BOWEN FLUORESCENCE MECHANISM FOR SPHERICALLY SYMMETRIC PLANETARY NEBULAE

R.Wehrse and A. Peraiah

Institut für Theoretische Astrophysik

Heidelberg, Germany

The transfer equation for the interlocked HeII Lya/OIII $2 \mathrm{p}^{2} \mathrm{P}_{2}$ $2 \mathrm{p} 3 \mathrm{~d} \mathrm{P}_{2}{ }^{\circ}$ lines is solved for spherical symmetry and for comparison in plane parallel approximation in the framework of the discrete space theory of radiative transfer. We used Harrington's ionization model (1969) without velocity fields and applied the partial redistribution function $\mathrm{R}_{\mathrm{IIA}}$ for both lines.

Appreciable differences occur between the two cases. In spherical symmetry the lines are narrow in the outer regions of the nebula, but much stronger than for plane parallel geometry so that more photons 
escape directly. Therefore, the efficiency of the Bowen conversion is reduced.

In addition it is found that the inner boundary condition is less important for spherical geometry than for plane parallel geometry as a consequence of the peaking effect.

This work has been performed as part of the program of the Sonderforschungsbereich 132 "Theoretische und Praktische Stellarastronomie", which is sponsored by the Deutsche Forschungsgemeinschaft.

\section{MODELS OF PLANETARY NEBULAE}

M. Perinotto

Astrophysical Observatory of Arcetri, Italy

From existing models of planetary nebulae an important discrepancy comes out with the low ionization potential ions, as $\mathrm{N}^{+}$and $\mathrm{O}^{+}$, whose calculated line intensities are smaller than the observed ones. In this work the effect of charge transfer reactions between single, double, and triple ionized oxygen and nitrogen with neutral hydrogen is taken into account in a model of NGC 7662. The ionization structure of oxygen and nitrogen becomes strongly modified. The computed fractional abundances of $0^{+}$and $\mathrm{N}^{+}$turn out substantially larger than in previous models, thus helping to reconcile the computed line emissions from low ionization potential ions with the observed ones. (Paper will appear in Astronomy and Astrophysics.) 